\title{
COREA GRAVÍDICA: REPORTE DE CASO Y REVISIÓN DE LA LITERATURA
}

\section{Chorea gravidarum: Case report and review of the literature}

\author{
Laura Juliana Rengifo-Quintero ${ }^{* 1}$; Mónica Andrea Beltrán-Avendaño, $M D^{2}$ \\ Recibido: septiembre 22/18 - Aceptado: octubre 22/19
}

\section{RESUMEN}

Objetivos: presentar un caso de corea gravídica y hacer una revisión sistemática de la literatura publicada sobre el tratamiento y pronóstico maternofetal de la entidad.

Materiales y métodos: se presenta el caso de una paciente de 16 años, primigesta, que ingresa a un hospital público de tercer nivel de complejidad, con 8 semanas de gestación, con clínica de 3 días de evolución caracterizada por movimientos involuntarios de la cabeza y las extremidades, con hiperreflexia en miembro inferior derecho. Antecedente de Corea de Sydenham. Se da tratamiento con antisicóticos y benzodiacepinas para la sintomatología y etiológico con penicilina benzatínica y control del cuadro a las 14 semanas. Suspendió el tratamiento a las 35 semanas. Parto normal a las 39 semanas. Se realizó una búsqueda en la base de datos de Medline vía PubMed, UptoDate, Medscape y Google Académico empleando los términos: "Pregnancy" and "Chorea

* Correspondencia: Laura Juliana Rengifo Quintero, teléfono: 3134217617, Dirección: Calle 90 \# 20 - 53, Barrio Diamante 2, Bucaramanga. e-mail: laurita_rengifo9307@hotmail.com

1 Estudiante de Medicina, Universidad Industrial de Santander, Bucaramanga (Colombia).

2 Ginecobstetra; especialista en Medicina Materno-Fetal. Docente del Departamento de Ginecobstetricia, Universidad Industrial de Santander, Unidad de Medicina Materno-Fetal del Hospital Universitario de Santander, Bucaramanga (Colombia).
Gravidarum". La búsqueda se limitó a reportes y series de casos o artículos de revisión, desde el año 2000 al 2019.

Resultados: se encontraron siete reportes de casos y una revisión de tema. El tratamiento se realizó con haloperidol benzodiacepinas y clorpromacina en cuatro de los siete casos. En uno de los dos casos con antecedentes de corea de Sydenham se utilizó penicilina. El pronóstico materno-fetal fue bueno en seis de siete casos, en un caso hubo restricción de crecimiento intrauterino.

Conclusión: el tratamiento de la corea gestacional principalmente es expectante y su finalidad es disminuir la sintomatología, el pronóstico maternofetal es bueno.

Palabras clave: embarazo; corea gravidarum; enfermedades del sistema nervioso.

\section{ABSTRACT}

Objectives: To present a case of chorea gravidarum and conduct a review of the published literature on the treatment for this condition, and on maternal and fetal prognosis.

Materials and methods: Case presentation of a 16-year-old primiparous patient admitted to a Level III public hospital at 8 weeks of gestation complaining of involuntary head and limb movements and right lower limb hyperreflexia 
lasting three days. The patient had a history of Sydenham chorea. Treatment with antipsychotics and benzodiazepines was given to manage symptoms, and with benzathine penicillin to address the etiology, achieving control at 14 weeks. Treatment was discontinued at 35 weeks and the patient went on to have normal delivery at 39 weeks. A search was conducted in the Medline via PubMed, UptoDate, Medscape and Google Scholar databases using the terms "Pregnancy and Chorea Gravidarum". The search was limited to case reports and case series or review articles published between 2000 al 2019. Results: Seven case reports and one review of the topic were found. In 4 of the 7 cases, treatment was based on haloperidol, benzodiazepines and chlorpromazine. Penicillin was used in one of two cases with a history of Sydenham chorea. Maternal and fetal prognosis was good in 6 of 7 cases, there was 1 case of intrauterine growth restriction.

Conclusion: Treatment of gestation chorea is primarily expectant and the goal is to reduce symptoms. Maternal and fetal prognosis is good.

Key words: Pregnancy; chorea gravidarum; nervous system diseases.

\section{INTRODUCCIÓN}

La corea gravídica o corea gestacional (CG) es un trastorno del movimiento durante la gestación, que cursa con movimientos espasmódicos involuntarios e incapacidad para mantener el cuerpo en una posición estable, estos movimientos se resuelven cuando finaliza el embarazo y ocurre en uno de cada 2000 a 3000 embarazos (1). Por lo general, se presenta en mujeres jóvenes y caucásicas. El 80\% de los casos se presenta en la primera gestación, el $50 \%$ debuta en el primer trimestre y el $60 \%$ de las gestantes refiere un antecedente de corea y, en algunos casos, hay recurrencia del cuadro en futuros embarazos (2). Las principales causas de CG son: fiebre reumática, proteína amiloide A sérica (serum amiloyd A [SSA]), enfermedad de Moyamoya, sífilis, síndrome antifosfolípidos y fármacos inductores de corea tales como: opioides, simpaticomiméticos, anticonceptivos orales, entre otros (3).

Independientemente de la etiología de la corea, todas generan una disfunción en la vía motora que comunica la corteza prefrontal con estructuras subcorticales. Esta vía motora tiene dos funciones, la primera consiste en la planeación e inicio del movimiento (vía directa); la segunda consiste en la inhibición de la respuesta motora (vía indirecta). En el caso de las coreas se ve comprometida la vía directa, lo que desencadena cuadros de hipercinesia por disminución de los potenciales inhibitorios (4). No se conoce el mecanismo exacto por el cual el embarazo desencadena o exacerba la corea, la hipótesis más aceptada es la que sugiere que los estrógenos y las hormonas progestacionales pueden llegar a sensibilizar los receptores dopaminérgicos (a nivel estriatal) e inducir la corea sobre todo en aquellas mujeres con patología preexistente a nivel de los ganglios basales (2).

En cuanto al diagnóstico de la CG se realiza el mismo enfoque de las coreas en población no gestante: historia clínica, examen neurológico, paraclínicos y neuroimágenes. Clínicamente se presenta con brazos hipotónicos oscilantes, la muñeca y los dedos asumen forma de tenedor con abducción del pulgar, la lengua entra y sale descontrolablemente (signo de la serpiente). Todos estos movimientos son rápidos, involuntarios, irregulares, espasmódicos y fluyen de manera aleatoria de una parte del cuerpo a otra. Las situaciones de estrés exacerban el cuadro, y los movimientos desaparecen durante el sueño (2). Los exámenes de laboratorio y las imágenes diagnósticas se orientan a buscar la causa etiológica básica, que puede ser: autoinmune, posinfecciosa, metabólica, vascular o farmacológica (5).

El tratamiento de la CG es principalmente expectante y su finalidad es disminuir la sintomatología, la morbilidad y las complicaciones (4). El tratamiento farmacológico tiene como objetivos: la disminución de la sintomatología y tratar el agente etiológico de la CG. El manejo sintomático se basa 
en tres grupos de fármacos: los antipsicóticos, las benzodiacepinas y los agentes que disminuyen la dopamina; se debe complementar con el tratamiento etiológico (6). Aunque en general el pronóstico de la CG es bueno, en algunos casos severos de CG puede llegar a presentarse hipertermia y la muerte (7).

La CG es una condición poco frecuente de la cual se dispone de información, por lo que es importante que los profesionales que se ocupan de la salud materna conozcan tanto el diagnóstico como el tratamiento y el pronóstico. El objetivo de este artículo es hacer una revisión de la literatura sobre el tratamiento de esta condición y el pronóstico materno y fetal.

\section{REPORTE DE CASO}

Paciente de 16 años, gesta 1, partos 0, abortos 0, con 8 semanas de gestación, con clínica de 3 días de evolución caracterizada por movimientos involuntarios de la cabeza y las extremidades, más hiperreflexia en miembro inferior derecho, que ingresa al servicio de urgencias del Hospital Universitario de Santander, institución pública de mediana y alta complejidad que atiende pacientes del régimen subsidiado por el Estado en el Sistema General de Seguridad Social en Colombia. Presentaba como antecedente un episodio de corea de Sydenham en la niñez, sin secuelas. No tomaba ningún medicamento, negaba consumo de sustancias psicoactivas o exposición a tóxicos. Fue valorada por neurología, quienes refieren que la paciente estaba alerta, con movimientos rápidos e involuntarios en extremidades, cabeza y tronco, destacándose el signo de la serpiente, el signo pronador de Wilson y el signo del "ahorcado" en miembros inferiores. Se considera que el cuadro es compatible con exacerbación de corea por estado de gravidez. Se inició manejo con clonazepam $0,5 \mathrm{mg} / 8 \mathrm{~h}$, ácido fólico $1 \mathrm{mg} /$ día, se toma en cuenta la contraindicación del uso de ácido valproico por riesgo de teratogenicidad. Los exámenes paraclínicos mostraron perfil infeccioso negativo, anticuerpos antifosfolípidos negativos, anticuerpos antiestreptolisinas negativos, electroen- cefalograma normal, tomografía cerebral simple en rangos de normalidad, ecografía obstétrica sin alteraciones.

Ante persistencia de los síntomas se administran $2 \mathrm{mg}$ de midazolam y 0,5 mg de haloperidol, con mejoría parcial, por lo que se incrementa la dosis de clonazepam a $3 \mathrm{mg} / 8 \mathrm{~h}$, manejo con el cual al tercer día de hospitalización se logra controlar en su totalidad los movimientos y se da egreso de la institución con manejo ambulatorio con clonazepam $0,5 \mathrm{mg} / 8 \mathrm{~h}$ y control por neurología. A los 9 días reingresa paciente con embarazo de 11 semanas y movimientos involuntarios por lo que se rehospitaliza. Se reinicia clonazepam $0,5 \mathrm{mg} / 8 \mathrm{~h}$ que toma durante 6 días, con mejoría parcial por lo que se incrementa la dosis de clonazepam a $3 \mathrm{mg} / 8$ h más carbamazepina $100 \mathrm{mg} / 8 \mathrm{~h}$ con aumento progresivo de dosis en 3 días a $200 \mathrm{mg} / 8 \mathrm{~h}$ con lo que se logra control de los movimientos anormales. Al día 13 de hospitalización realizan junta médica de perinatología que indica iniciar profilaxis antibiótica con penicilina benzatínica 2`400.000 U debido a su antecedente médico. Por mejoría se da salida a las 14,2 semanas de embarazo con disminución progresiva del clonazepam y se indica continuar con carbamazepina. Ingresa de nuevo a las 39 semanas de gestación con actividad uterina irregular y asintomática para corea, a pesar de haber suspendido la medicación hacía un mes; un monitoreo fetal mostró buen bienestar del feto. Se hace diagnóstico de preparto, se reinicia carbamazepina de $200 \mathrm{mg} / 8 \mathrm{~h}$; posteriormente, tiene parto por vía vaginal, con recién nacido vivo femenino con peso de $2910 \mathrm{~g}$, talla $50 \mathrm{~cm}$, perímetro torácico: $33 \mathrm{~cm}$, perímetro cefálico: $35 \mathrm{~cm}$, APGAR: 8 al min - 9 a los $5 \mathrm{~min}, \mathrm{y}$ buena adaptación neonatal. La paciente y el recién nacido presentaron una evolución satisfactoria y se da egreso a las 24 horas.

Aspectos éticos. Se solicitó autorización a la paciente para la publicación del reporte de caso. Se garantizó la confidencialidad de la información y la privacidad de los datos de la paciente. 


\section{MATERIALES Y MÉTODOS}

Con base en la pregunta: ¿cuál es el tratamiento para la CG?, se realizó una búsqueda en la base de datos de Medline vía PubMed empleando los siguientes términos: "Pregnancy" and "Chorea Gravidarum". La búsqueda se limitó por los siguientes filtros: "Case Reports”, "Review”, “Systematic Reviews”, “Humans", "Spanish and English", desde el año 2000 al 2019. Se seleccionaron estudios que incluyeran mujeres gestantes con corea; se excluyeron editoriales, reseñas, cartas al editor, artículos duplicados y estudios que reportaran toxicidad por uso de fármacos inductores de corea. Se extrajo información sobre el tratamiento y pronóstico de la CG. La búsqueda se complementó con otras bases de datos como UptoDate, Medscape y Google Académico. El proceso de selección fue realizado por una de las autoras, la cual inicialmente leyó los resúmenes de cada artículo y seleccionó aquellos que cumplían los criterios de inclusión y exclusión. Posteriormente obtuvo los textos completos de los estudios seleccionados y extrajo la información sobre el tratamiento empleado. Se obtuvo información respecto a: tipo de estudio, número de pacientes incluidas, número de episodios, país de procedencia, manejo farmacológico instaurado, comorbilidades, complicaciones fetales o maternas. Se realizó análisis narrativo de los hallazgos.

\section{RESULTADOS}

Inicialmente se obtuvieron 32 títulos, al aplicar los criterios de selección quedaron 8 títulos, de las cuales 7 correspondieron a reportes de caso (1, 2, 8-12) y una revisión de tema (13). Estos reportes resumen la información de 7 pacientes en gestación con edades entre los 19 y 28 años, la etiología de la corea fue asociada a fiebre reumática $(8,9)$, enfermedad de Moyamoya (2) y mutación del factor V de Leiden (10); la edad gestacional de presentación varió entre 8 y 40 semanas, con una mayor frecuencia en el primer trimestre (tabla 1). En un estudio publicado en el 2003 no fue posible obtener el texto completo (14).
El tratamiento instaurado fue sintomático con haloperidol $(1,10,11)$ y benzodiacepinas más clorpromazina (12) con buen control de los movimientos. El tratamiento de la causa básica con penicilina cristalina se dio en un caso (8). En otro caso asociado a fiebre reumática no se utilizó tratamiento para estreptococo grupo A ( ). Ramachandran et al. (13) describen que los medicamentos más utilizados en el embarazo son el haloperidol, clorpromazina, risperidona y pimozida (categoría C). Refieren que algunos autores los suspenden 5 a 10 días antes del parto para minimizar los síntomas extrapiramidales en el neonato. En segundo lugar mencionan los anticonvulsivantes para el manejo de los espasmos musculares graves y los movimientos tónico-clónicos. Los recomendados durante la gestación son: fenobarbital y carbamazepina; aunque mencionan como alternativa ácido valproico, no se encontró en este estudio ningún caso en que se utilizara. En tercer lugar señalan las benzodiacepinas (13). Sin embargo, estas últimas no están recomendadas en el embarazo a no ser que sea estrictamente necesario, pues estos fármacos atraviesan la barrera placentaria y generan alteraciones a nivel fetal, principalmente durante el primer trimestre (hendidura palatina o labio leporino, alteraciones cardiacas, etc.) y el tercer trimestre (depresión neurológica del recién nacido, trastornos de regulación térmica, apnea, APGAR disminuido, entre otros); en caso de que deban ser usadas se recomiendan las de vida media biológica corta (15).

Respecto a las complicaciones maternas se describe que una paciente presentó infarto cerebral materno (10) y otro caso se asoció a ruptura prematura de membranas (12); en cuanto a las perinatales se describe un caso de restricción de crecimiento intrauterino (1). La CG se resolvió al terminar la gestación, sin secuelas, excepto en un caso en el que se presentaron secuelas de persistencia de leve movimiento en mano derecha (12). No se encontraron informes de mal pronóstico fetal o materno. 


\begin{tabular}{|c|c|c|c|c|c|}
\hline Autor & $\begin{array}{l}\text { Etiología } \\
\text { de la CG }\end{array}$ & $\begin{array}{l}\text { Características } \\
\text { de la paciente }\end{array}$ & Tratamiento & Complicaciones & $\begin{array}{c}\text { Evolución de la } \\
\text { paciente }\end{array}$ \\
\hline $\begin{array}{l}\text { Palanivelu LM, } \\
\quad 2007 . \\
\text { Reino Unido (1) }\end{array}$ & Desconocida & $\begin{array}{l}20 \text { años, G1P0, embarazo de } 16 \\
\text { semanas, sin antecedentes de im- } \\
\text { portancia }\end{array}$ & Haloperidol & $\begin{array}{c}\text { Feto con restricción } \\
\text { del crecimiento (no } \\
\text { se establece si está } \\
\text { asociado a la CG) }\end{array}$ & $\begin{array}{l}\text { Cuadro se resolvió } \\
\text { favorablemente, sin } \\
\text { secuelas en la madre; } \\
\text { recién nacido con } \\
\text { restricción del } \\
\text { crecimiento }\end{array}$ \\
\hline $\begin{array}{l}\text { A Kim et al., } \\
\text { 2009. Corea del } \\
\text { Sur (2) }\end{array}$ & $\begin{array}{c}\text { Enfermedad de } \\
\text { Moyamoya }\end{array}$ & $\begin{array}{l}28 \text { años, G1P0, embarazo de } 8,6 \\
\text { semanas, sin antecedentes de im- } \\
\text { portancia }\end{array}$ & No reportan & Ninguna & $\begin{array}{c}\text { El cuadro se resolvió } \\
\text { favorablemente y } \\
\text { no quedó ninguna } \\
\text { secuela y RN sin } \\
\text { alteraciones }\end{array}$ \\
\hline $\begin{array}{l}\text { Pathania et al., } \\
\text { 2013. India (8) }\end{array}$ & Fiebre reumática & $\begin{array}{l}21 \text { años, G1P0, embarazo } 32,4 \\
\text { semanas y antecedente de episo- } \\
\text { dio de fiebre reumática }\end{array}$ & $\begin{array}{l}\text { Penicilina } \\
\text { benzatínica }\end{array}$ & Ninguna & No reportan \\
\hline $\begin{array}{l}\text { Ghike et al., } \\
\text { 2007. India (9) }\end{array}$ & Fiebre reumática & $\begin{array}{l}22 \text { años, G2P1, embarazo de } 8,1 \\
\text { semanas, antecedente de corea } \\
\text { de Sydenham en la niñez }\end{array}$ & No reportan & Ninguna & No reportan \\
\hline $\begin{array}{l}\text { Qasim A, } 2000 . \\
\text { Reino Unido } \\
\quad(10)\end{array}$ & $\begin{array}{l}\text { Mutación del } \\
\text { factor V de } \\
\text { Leiden de la } \\
\text { coagulación }\end{array}$ & $\begin{array}{l}24 \text { años, G1P0, embarazo } 10 \\
\text { semanas, sin antecedentes de im- } \\
\text { portancia }\end{array}$ & $\begin{array}{l}\text { Haloperidol } 1 \\
\text { mg } 3 \text { tab. día } \\
\text { más heparina } \\
\text { endovenosa }\end{array}$ & $\begin{array}{l}\text { Infarto cerebral } \\
\text { extenso }\end{array}$ & No reportan \\
\hline $\begin{array}{l}\text { Karageyin et al., } \\
\text { 2002. Turquia } \\
\text { (11) }\end{array}$ & Desconocida & $\begin{array}{l}19 \text { años, G1P0, con embarazo } \\
\text { de } 40 \text { semanas y fumadora de } 4 \\
\text { paquetes de cigarrillo diarios, sin } \\
\text { antecedentes de importancia }\end{array}$ & $\begin{array}{c}\text { Haloperidol } 1 \\
\text { mg día }\end{array}$ & No reporta & $\begin{array}{c}\text { Cuadro se resolvió } \\
\text { favorablemente, sin } \\
\text { secuelas en la madre, } \\
\text { con un recién nacido } \\
\text { normal }\end{array}$ \\
\hline $\begin{array}{l}\text { Fernando } \\
\text { Germes Piña, } \\
\text { 2009. México } \\
\text { (12) }\end{array}$ & Desconocida & $\begin{array}{l}22 \text { años, G3P1A1, embarazo de } \\
36,1 \text { semanas, sin antecedentes } \\
\text { de importancia }\end{array}$ & $\begin{array}{c}\text { Diazepam y } \\
\text { clorpromazina }\end{array}$ & $\begin{array}{l}\text { Ruptura prematura } \\
\text { de membranas }\end{array}$ & $\begin{array}{l}\text { Secuela de un leve } \\
\text { movimiento en la } \\
\text { mano derecha y } \\
\text { recién nacido sin } \\
\text { alteraciones }\end{array}$ \\
\hline
\end{tabular}

\section{CONCLUSIONES}

El tratamiento de la CG principalmente es expectante y su finalidad es disminuir la sintomatología. El pronóstico materno-fetal es bueno.

\section{REFERENCIAS}

1. Palanivelu LM. Chorea gravidarum. J Obstet Gynaecol. 2007;27(3):310. https://doi.org/10.1080 /01443610701241134
2. Kim A, Choi $\mathrm{CH}$, Han $\mathrm{CH}$, Shin JC. Consecutive pregnancy with chorea gravidarum associated with moyamoya disease. J Perinatol. 2009;29(4):317-9. https://doi.org/10.1038/jp.2008.183

3. Fenichel GM. Movement Disorders. En: Fenichel GM, editor. Clinical Pediatric Neurology. A Signs and Symptoms Approach. 6 ed. Nashville: Elsevier; 2009. p 293-311. https://doi.org/10.1016/B978-1-41606185-4.00014-9 
4. Cardoso F, Seppi K, Mair KJ, Wenning GK, Poewe W. Seminar on Choreas. Lancet Neurol. 2006;5(7):589-602. https://doi.org/10.1016/S1474-4422(06)70494-X

5. Hermann A, Walker RH. Diagnosis and treatment of chorea syndromes. Curr Neurol Neurosci Rep. 2015;15(2):514. https://doi.org/10.1007/s11910014-0514-0

6. Yilmaz S, Mink JW. Treatment of Chorea in Childhood. Pediatr Neurol. 2019 Sep 7. pii: S08878994(19)30817-3. https://doi.org/10.1016/j. pediatrneurol.2019.08.013

7. Ichikawa K, Kim RC, Givelber H, Collins GH. Chorea gravidarum. Report of a fatal case with neuropathological observations. Arch Neurol. 1980;37(7):429-32. https://doi.org/10.1001/archneur. 1980.00500560059007

8. Pathania M, Upadhyaya S, Lali BS, Sharma A. Chorea gravidarum: A rarity in West still haunts pregnant women in the East. BMJ Case Rep. 2013; pii: bcr2012008096. https://doi.org/10.1136/bcr-2012008096

9. Ghike S, Gawande M, Jain S. A rare case of chorea gravidarum. South Asian Federation of Obstetrics and Gynecology. 2010 [visitado 2017 abr 9];2(2):145-6. https://doi.org/10.5005/jp-journals-10006-1084

10. Qasim A. An unusual case of chorea gravidarum. Postgrad Med J. 2000;76:374-8. https://doi.org/10.1136/ pmj.76.896.374
11. Karageyim AY, Kars B, Dansuk R, Aygun E, Unal O, Turan MC. Chorea gravidarum: A case report. J Matern Fetal Neonatal Med. 2002;12(5):353-4. https://doi.org/10.1080/jmf.12.5.353.354

12. Germes-Piña F. Corea gravídica. Reporte de un caso. Ginecol Obstet Mex. 2009 [visitado 2017 abr 9];77(3):156-9. Disponible en: http:// www.medigraphic.com/cgi-bin/new/resumen. cgi? IDARTICULO $=26927$

13. Ramachandran TS, Talavera F, Galvez-Jimenez N, Benbadis SR, Gancher ST. Chorea Gravidarum. New York: Medscape; 2017. Disponible en: http://emedicine.medscape.com/article/1149725-overview

14. Fam NP, Chisholm RJ. Chorea in a pregnant woman with rheumatic mitral stenosis. Can J Cardiol. 2003;19(6):719-21.

15. Sosa L. Farmacoterapia durante el embarazo. Arch Med Int. 2009;31(4):87-92.

\section{CONTRIBUCIÓN DE LOS AUTORES}

Laura Juliana Rengifo-Quintero: búsqueda sistemática de la literatura, redacción del manuscrito. Mónica Andrea Beltrán-Avendaño: caso clínico, supervisión y corrección de la redacción del manuscrito. 